\title{
Metadherin in prostate, bladder, and kidney cancer: A systematic review
}

\author{
ZHAO WANG $^{1 *}$, YONG-BAO WEI $^{1 *}$, YUN-LIANG GAO $^{1}$, BIN YAN $^{1}$, JIN-RUI YANG $^{1}$ and QIONG GUO $^{2}$ \\ ${ }^{1}$ Department of Urology, The Second Xiangya Hospital, Central South University, Changsha, Hunan 410011; \\ ${ }^{2}$ Department of Urology, The Third Hospital of Changsha, Changsha, Hunan 410015, P.R. China
}

Received July 2, 2014; Accepted August 11, 2014

DOI: $10.3892 / \mathrm{mco} .2014 .392$

\begin{abstract}
Metadherin (MTDH) has been identified as an important oncogene in carcinogenesis, tumor progression and metastasis in numerous malignancies, through signal transduction pathways. MTDH is a potential biomarker and therapeutic target in cancers. The present systematic review was performed to search for studies regarding MTDH and prostate, bladder and kidney cancer using several databases and the eligible studies were reviewed. MTDH expression was found to significantly increase in prostate, bladder and kidney cancers, not only in clinical tissue samples, but also in cancer cell lines. Reviewing the clinical and statistical analysis revealed that MTDH may be involved in urologic cancer progression, metastasis and prognosis. MTDH may be an independent or one of the cofactors in urologic cancers for prediction of patient survival, and may be involved in potential anticancer strategies. MTDH may be associated with several signal transduction pathways in urologic cancers, indicating latent targets to develop anticancer therapeutic strategy. Further studies are required to confirm these findings.
\end{abstract}

\section{Introduction}

Metadherin (MTDH), also known as astrocyte-elevated gene (AEG)-1 and lysine-rich CEACAM-1-associated protein (LYRIC) (1-2), was first identified in human fetal astrocytes induced by human immunodeficiency virus-1 in 2002 (3). MTDH has been cloned for nearly ten years (4-6) and is considered to be an important oncogene in carcinogenesis,

Correspondence to: Professor Jin-rui Yang, Department of Urology, The Second Xiangya Hospital, 139 Renmin Road, Central South University, Changsha, Hunan 410011, P.R. China

E-mail: 2xiangya2010@gmail.com

Dr Qiong Guo, Department of Urology, The Third Hospital of Changsha, 176 Laodong Xi Road, Changsha, Hunan 410015, P.R. China

E-mail: bigstrongman2005@163.com

*Contributed equally

Key words: metadherin, prostate, bladder, kidney, cancer tumor progression and metastasis, via a series of signaling pathways (7-9). However, as the exact functions of MTDH in cancer remain unclear, particularly in malignant tumors, future investigations are required (10).

Recent studies have shown that high expressions of MTDH are present in various tumors, involving the nervous, urogenital, respiratory and digestive systems (9,11-19). Evidence indicates that MTDH knockdown significantly inhibited the growth of gastric cancer cells (9). MTDH is an anti-apoptotic gene in glioma cells and could be used as a target of microRNA-136 in anticancer therapeutic strategy (11). A study revealed that MTDH promotes invasion and metastasis through the activation of nuclear factor- $\kappa \mathrm{B}(\mathrm{NF}-\kappa \mathrm{B})$, interleukin- 8 and matrix metalloproteinase-9 in breast cancer (12), which is consistent with studies in non-small cell lung cancer and glioma $(13,14)$. MTDH has been shown to be associated with a poor prognosis in malignancies, including non-small cell lung cancer, invasive breast cancer, laryngeal squamous cell carcinoma, gallbladder adenocarcinoma, tongue carcinoma and bladder cancer (15-19). MTDH is also associated with the prognosis of carcinoma, possibly due to chemoresistance and radiosensitivity in cancer therapy $(20,21)$.

Increasing evidence indicates that MTDH is an important mediator in signal transduction pathways in malignancies, a potential biomarker to predict cancer survival and provides information to develop target chemotherapies. The present systematic review aimed to investigate the expression, pathogenesis and therapeutic strategies of MTDH in prostate, bladder and kidney cancers.

\section{Materials and methods}

Literature selection. The inclusion criteria for the search are any studies regarding MTDH and prostate, bladder and kidney cancer. The exclusion criteria are as followings: i) Duplicate or series reporting, in which the most detailed results were included; ii) conference report, review or letter; and iii) study language not in English or Chinese.

Literature search. A comprehensive literature search of the English and Chinese databases was performed from the time when establishing the databases to 25 March, 2014, using Pubmed, Highwire, Springerlink, EBSCO, ProQuest, Wiley, Google Scholar, China National Knowledge Infrastructure 
and Wanfang Data Knowledge Service Platform (Wanfang data). The studies regarding MTDH and prostate, bladder and kidney cancer respectively were searched for. The following example is the search strategy of MTDH and prostate cancer in Pubmed: (metadherin or MTDH or astrocyte elevated gene-1 or AEG-1 or lysine-rich CEACAM1 or LYRIC) and (prostate or prostate neoplasm or prostate cancer). The references of the associated studies were checked manually to avoid the loss of relevant studies. Approximately 537 studies were consistent with the search strategies in these databases, and 13 were eligible for the search. Fig. 1 shows a flowchart of the search process. The detailed search results of MTDH and prostate, bladder and kidney cancers are listed in Table I.

\section{Results and Discussion}

MTDH and prostate cancer. A total of 8 eligible studies reported the association between MTDH and prostate cancer. High MTDH expression was detected in tissue specimens and prostate cancer cell lines, and was confirmed to be associated with prognosis and patient survival. Studies also investigated the possible signal transduction pathways in prostate cancer cell lines mediated by MTDH, which may be potential therapeutic targets for anticancer.

MTDH overexpression in prostate cancer. One study showed that MTDH overexpression in prostate cancer was $66.7 \%$ (22), whereas in another study, the total expression of MTDH was $100 \%$ (23). The later case-control study involved 20 prostate cancer samples from radical prostatectomy and 20 benign prostatic hyperplasia (BPH) specimens from transurethral resection. MTDH strongly positive expression in prostate cancer was $80 \%$, whereas it was $10 \%$ in BPH. Weakly positive expression of MTDH in prostate cancer was reduced to only $20 \%$, but $35 \%$ in BPH. The negative expression in BPH reached $55 \%$.

A high expression of MTDH in prostate cancer cell lines was also observed in the eligible studies. Quantitative reverse transcription polymerase chain reaction (RT-PCR) and western blotting showed that the expression level of MTDH in prostate cancer cells was nearly three times higher compared to those in non-cancerous human prostatic epithelial cell line (RWPE-1 cells) at the mRNA and protein level, respectively. High MTDH expressions were found in three common prostate cancer cells linesl: LNCaP, DU145 and PC-3 cell lines $(23,24)$.

Thirkettle et al (10) reported the MTDH distribution in the subcellular compartments of prostate cancer and benign samples. The MTDH expression in the nucleus of luminal cells was much higher in benign cases $(82.5 \%)$ compared to in tumors (26.6\%). The distributions of MTDH in cytoplasm alone, cytoplasm plus nucleus and global cells in prostate cancer samples were $33.6 \%, 42.9 \%$ and $8.8 \%$, respectively. The distribution of MTDH was different in subcellular compartments, which was also associated with tumor grades (10).

These results show that an association exists between the expression of MTDH and prostate cancer. MTDH overexpression not only occurs in human tissue samples, but also in prostate cancer cell lines. Studies have demonstrated that the

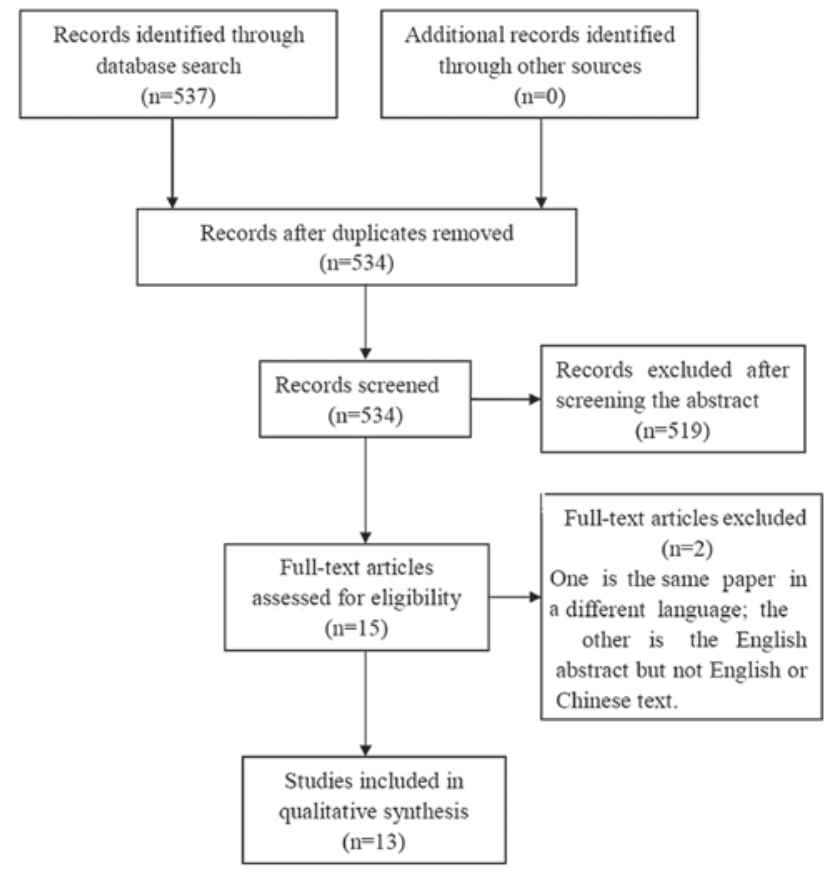

Figure 1. A flowchart of the search process regarding metadherin in prostate, bladder and kidney cancer.

high expression of MTDH is similar in prostate cancer, but is not distinctive in benign lesions (23). However, in another study, MTDH was also proved to be highly expressed in benign samples (10).

Progression and survival of MTDH in prostate cancer. A follow-up of 50 patients for $<120$ months indicated that mean survival times in patients with MTDH in the nucleus are longer than those losses of MTDH in the nucleus (10). In patients with bone metastasis, a high expression of MTDH was confirmed in prostate bone metastases $(81.8 \%)$ and it was mainly distributed in the cytoplasm and membrane (10). Following an investigation of 97 radical prostatectomy samples, Erdem et al (22) showed that MTDH combined with the basic fibroblast growth factor is one of the independent prognostic parameters. These results indicate that MTDH may be a potential prognostic factor of prostate cancer.

MTDH and signal transduction in prostate cancer. The pathogenesis of MTDH in tumors may be interpreted as participating in signal transduction pathways, including $\mathrm{NF}-\kappa \mathrm{B}, \mathrm{Ha}-\mathrm{ras}$ and AKT. These signaling pathways may be involved in carcinogenesis, metastasis and progression in malignancies, predicting that MTDH may be a biomarker in cancers $(7,25,26)$.

Ash et al (27) used the yeast two-hybrid assay to identify that BRCA2 and CDKN1A interacting protein (BCCIP) is an associated protein of MTDH. In DU145 cells, MTDH was found to be a negative regulator of BCCIP $\alpha$, which induces DU145-apparent neuroendocrine differentiation. The NF- $\kappa \mathrm{B}$ signaling pathway was considered to be involved in the interactions of MTDH and BCCIP in prostate cancer cells. Another study also verified that the knockdown of MTDH downregulated the activity of NF- $\mathrm{kB}$ in PC-3 and DU145 cells (23). In 


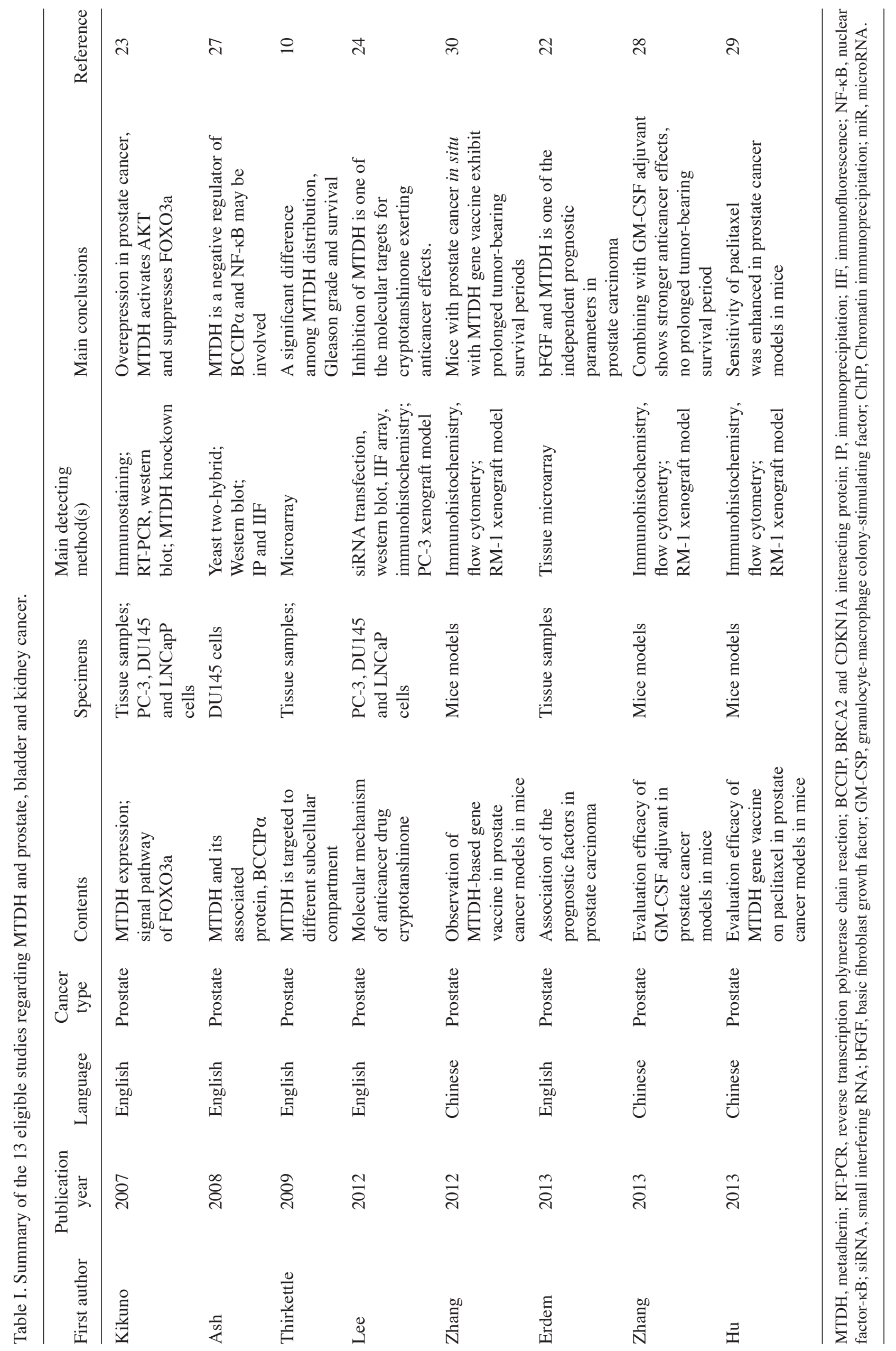




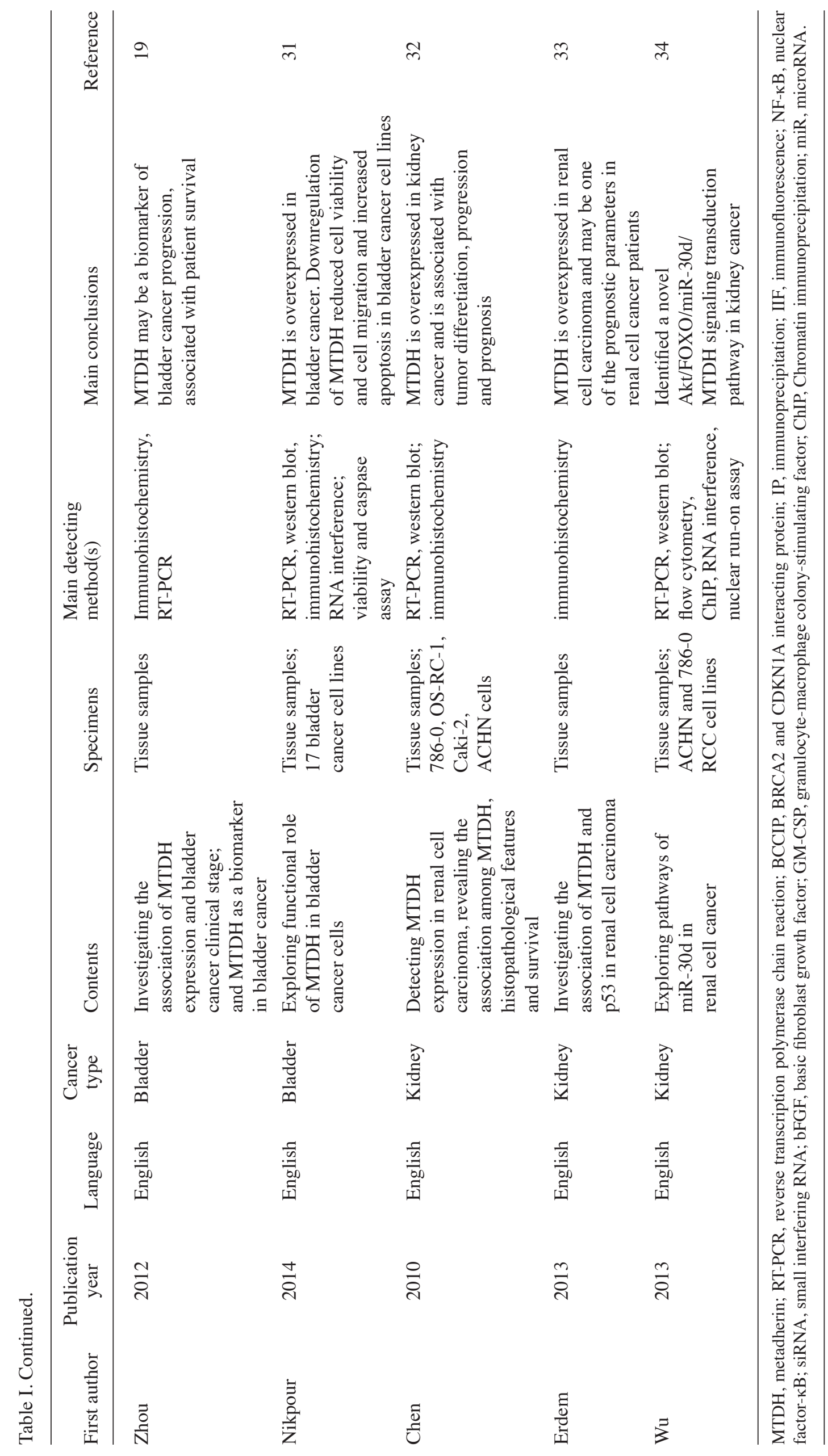


addition, these results also prove that the inhibition of prostate cancer progression by MTDH knockdown may be mediated by suppression of AKT and upregulation of FOXO3a activity (23). In hypoxic PC-3 cells, MTDH may participate in the downstream of the hypoxia-inducible factor $1 \alpha(\mathrm{HIF}-1 \alpha)$ or phosphoinositide 3-kinase pathways (24).

MTDH and prostate cancer therapies. The signaling pathways of MTDH were reviewed systematically in prostate cancer previously in this study and it was concluded that MTDH may be a target in the treatment of prostate cancer. In order to investigate the molecular mechanism of cryptotanshinone in anticancer, several genes were analyzed in the study by Lee et al (24), including MTDH and HIF-1 $\alpha$. Inhibition of MTDH expression was found to be one of the molecular targets for cryptotanshinone exerting anticancer effects (24).

Previous studies have examined MTDH-based DNA vaccines in prostate cancer using mice models (28-30). The results have shown that a significant enhancement of humoral and cellular immune responses was detected in MTDH-immunized mice. With the MTDH gene vaccine, mice with prostate cancer in situ exhibited prolonged tumor-bearing survival times (28). This was considered to be due to the inhibition of the proliferation and invasion of prostate cancer. The MTDH gene vaccine combined with granulocyte-macrophage colony-stimulating factor (GM-CSF) adjuvant shows stronger anticancer effects, except that there was no significant difference in tumor-bearing survival time (29). The MTDH gene vaccine combined with paclitaxel exhibits significant inhibition of prostate cancer growth and prolonged tumor-bearing survival times (30).

MTDH and bladder cancer. The first study of MTDH expression in bladder cancer was in 2012 by Zho et al (19). The study involved 15 benign cases and 60 cancer samples. The results showed a significant difference of MTDH expression between bladder benign specimens and cancers (19). This result was also proved by another study by detection of MTDH mRNA expression in bladder benign and cancer samples (31), and MTDH expression has also been verified in 17 bladder cancer cell lines (31). RT112 cell lines have the highest expression, which is higher than that of DU145 and LNCaP as the controls, whereas HT1376 showed the lowest expression in the 17 cell lines (31).

Another study indicated that MTDH is a potential prognostic factor of bladder cancer, as a significant association was demonstrated between the expression of MTDH and clinicopathological stage, tumor classification, tumor multiplicity and survival time (19). However, no significant correlations were reported in the study by Nikpour et al (31) between the expression of MTDH mRNA and bladder cancer grade, tumor category and lymph node metastasis.

As a positive correlation between MTDH and Ki67 has been demonstrated, MTDH may promote bladder cancer tissue growth (19). The study by Nikpour et al (31) also confirmed that MTDH promoted bladder cancer cell survival, clonogenicity and migration, which were verified by downregulation of MTDH via small interfering RNA. In addition to increased apoptosis, reductions of cell viability and migration have also been observed in bladder cancer (31).
MTDH and kidney cancer. MTDH overexpression in primary kidney cancer has been confirmed by immunohistochemistry, RT-PCR and western blot. Two studies found that the expression of MTDH in kidney carcinoma specimens was high compared to normal tissues $(32,33)$. An in vitro study by Chen et al (32) investigated the expression of MTDH in four kidney cancer cell lines; 786-0, OS-RC-1, Caki-2 and ACHN. A significantly higher level of MTDH was expressed in these four cell lines in the cytoplasm compared to the HK-2 cells as the control (32).

The results of Chen et al (32) indicated that the MTDH expression level is associated with the differentiation degree of renal cell carcinoma and clinical stage, with tumor and metastasis classifications, but not node classifications. Survival analysis confirmed a shorter survival time when the MTDH level was higher (32). The conclusion was also verified by another study showing that MTDH may be one of the prognostic parameters in renal cell cancer patients (33). A novel signal transduction pathway Akt/FOXO/miR-30d/MTDH in renal cell carcinoma was reported by the study by Wu et al (34), providing a new insight into the anticancer strategy of kidney cancer.

Limitations of the present studies and prospects. MTDH, as one of numerous oncogenes, has been studied in urogenital cancers for $<10$ years. Limitations of the current studies may exist in this area and are as follows.

Firstly, a larger tissue sample size should be analyzed to measure the association between MTDH expression and urogenital cancers in progression, metastasis and prognosis. Regarding MTDH and progression of bladder cancer, conflicting conclusions were reported in two studies $(19,31)$ possibly due to their limited samples. Secondly, follow-up times and repeated studies are required to investigate the higher expression of MTDH as an influence of prognostic factors. Only one study performed a 120-month follow-up of prostate cancer, which may not be enough (10). Follow-up information is not available in bladder and kidney cancers. Thirdly, increasing detection methods and more comprehensive sources of specimen should be tested in further studies. To confirm MTDH as a potential biomarker in urogenital cancers, except for body organs or tissues, samples of blood, plasma, urine, prostatic and seminal fluid may also be taken into consideration to detect the MTDH expression. Fourthly, in vitro and animal models are required to reveal the function of MTDH as an oncogene and its signaling transduction pathways. Signaling transduction pathways of MTDH in prostate and kidney cancers is limited in the current studies. An increase in the detailed signaling pathways should be further investigated to define the role of MDTH in tumor pathogenesis. Further studies in signaling may provide novel targets for anticancer therapies.

MTDH is overexpressed in a number of urologic cancers, including prostate, bladder and kidney cancers. MTDH may be involved in urologic cancer progression, metastasis and prognosis and may be associated with several signaling transduction pathways in urologic cancers, indicating latent targets to develop anticancer therapeutic strategies. Further studies are required to confirm the aforementioned conclusions. 


\section{Acknowledgements}

The present study was supported by the Fundamental Research Funds for the Central Universities of Central South University in 2013 (no. 2013zzts095).

\section{References}

1. Sarkar D and Fisher PB: AEG-1/MTDH/LYRIC: clinical significance. Adv Cancer Res 120: 39-74, 2013.

2. Lee SG, Kang DC, DeSalle R, et al: AEG-1/MTDH/LYRIC, the beginning: initial cloning, structure, expression profile, and regulation of expression. Adv Cancer Res 120: 1-38, 2013.

3. Su ZZ, Kang DC, Chen Y, et al: Identification and cloning of human astrocyte genes displaying elevated expression after infection with HIV-1 or exposure to HIV-1 envelope glycoprotein by rapid subtraction hybridization, RaSH. Oncogene 21 3592-3602, 2002.

4. Kang DC, Su ZZ, Sarkar D, et al: Cloning and characterization of HIV-1-inducible astrocyte elevated gene-1, AEG-1. Gene 353: $8-15,2005$

5. Britt DE, Yang DF, Yang DQ, et al: Identification of a novel protein, LYRIC, localized to tight junctions of polarized epithelial cells. Exp Cell Res 300: 134-148, 2004.

6. Sutherland HG, Lam YW, Briers S, et al: 3D3/lyric: a novel transmembrane protein of the endoplasmic reticulum and nuclear envelope, which is also present in the nucleolus. Exp Cell Res 294: 94-105, 2004

7. Emdad L, Sarkar D, Su ZZ, et al: Astrocyte elevated gene-1: recent insights into a novel gene involved in tumor progression, metastasis and neurodegeneration. Pharmacol Ther 114: 155-170, 2007.

8. Hu G, Wei Y and Kang Y: The multifaceted role of MTDH/AEG-1 in cancer progression. Clin Cancer Res 15: 5615-5620, 2009.

9. Huang W, Yang L, Liang S, et al: AEG-1 is a target of perifosine and is over-expressed in gastric dysplasia and cancers. Dig Dis Sci 58: 2873-2880, 2013.

10. Thirkettle HJ, Girling J, Warren AY, et al: LYRIC/AEG-1 is targeted to different subcellular compartments by ubiquitinylation and intrinsic nuclear localization signals. Clin Cancer Res 15: 3003-3013, 2009.

11. Yang Y, Wu J, Guan H, et al: MiR-136 promotes apoptosis of glioma cells by targeting AEG-1 and Bcl-2. FEBS Lett 586 3608-3612, 2012.

12. Zhao Y, Kong X, Li X, et al: Metadherin mediates lipopolysaccharide-induced migration and invasion of breast cancer cells. PLoS One 6: e29363, 2011.

13. Sun S, Ke Z, Wang F, et al: Overexpression of astrocyte-elevated gene- 1 is closely correlated with poor prognosis in human non-small cell lung cancer and mediates its metastasis through up-regulation of matrix metalloproteinase-9 expression. Hum Pathol 43: 1051-1060, 2012.

14. Liu L, Wu J, Ying Z, et al: Astrocyte elevated gene-1 upregulates matrix metalloproteinase-9 and induces human glioma invasion. Cancer Res 70: 3750-3759, 2010.

15. Tokunaga E, Nakashima Y, Yamashita N, et al: Overexpression of metadherin/MTDH is associated with an aggressive phenotype and a poor prognosis in invasive breast cancer. Breast Cancer 21 341-349, 2012

16. Liu Y, Su Z, Li G, et al: Increased expression of metadherin protein predicts worse disease-free and overall survival in laryngeal squamous cell carcinoma. Int J Cancer 133: 671-679, 2013.
17. Liu DC and Yang ZL: MTDH and EphA7 are markers for metastasis and poor prognosis of gallbladder adenocarcinoma. Diagn Cytopathol 41: 199-205, 2013.

18. Ke ZF, He S, Li S, et al: Expression characteristics of astrocyte elevated gene-1 (AEG-1) in tongue carcinoma and its correlation with poor prognosis. Cancer Epidemiol 37: 179-185, 2013.

19. Zhou J, Li J, Wang Z, et al: Metadherin is a novel prognostic marker for bladder cancer progression and overall patient survival. Asia Pac J Clin Oncol 8: e42-e48, 2012.

20. Li C, Li Y, Wang X, et al: Elevated expression of astrocyte elevated gene-1 (AEG-1) is correlated with cisplatin-based chemoresistance and shortened outcome in patients with stages III-IV serous ovarian carcinoma. Histopathology 60: 953-963, 2012.

21. Zhao Y, Moran MS, Yang Q, et al: Metadherin regulates radioresistance in cervical cancer cells. Oncol Rep 27: 1520-1526, 2012.

22. Erdem H, Yildirim U, Uzunlar AK, et al: Relationship among expression of basic-fibroblast growth factor, MTDH/astrocyte elevated gene-1, adenomatous polyposis coli, matrix metalloproteinase 9, and COX-2 markers with prognostic factors in prostate carcinomas. Niger J Clin Pract 16: 418-423, 2013.

23. Kikuno N, Shiina H, Urakami S, et al: Knockdown of astrocyte-elevated gene-1 inhibits prostate cancer progression through upregulation of FOXO3a activity. Oncogene 26: 7647-7655, 2007.

24. Lee HJ, Jung DB, Sohn EJ, et al: Inhibition of hypoxia inducible factor alpha and astrocyte-elevated gene-1 mediates cryptotanshinone exerted antitumor activity in hypoxic PC-3 cells. Evid Based Complement Alternat Med 2012: 390957, 2012.

25. Zhang J, Zhang Y, Liu S, et al: Metadherin confers chemoresistance of cervical cancer cells by inducing autophagy and activating ERK/NF-kappaB pathway. Tumour Biol 34: 2433-2440, 2013

26. Yu C, Liu Y, Tan $\mathrm{H}$, et al: Metadherin regulates metastasis of squamous cell carcinoma of the head and neck via AKT signalling pathway-mediated epithelial-mesenchymal transition. Cancer Lett 343: 258-267, 2014.

27. Ash SC, Yang DQ and Britt DE: LYRIC/AEG-1 overexpression modulates BCCIP alpha protein levels in prostate tumor cells. Biochem Biophys Res Commun 371: 333-338, 2008.

28. Zhang C, Li H, Qian B, et al: Metadherin/astrocyte elevated gene-1-based DNA vaccine suppresses progression and metastasis in prostate cancer of mice. Chin J Exp Surg 30: 2148-2151, 2013 (In Chinese)

29. Hu W: GM-CSF combined with vaccine of MTDH inhibiting prostate cancer growth and metastasis. Master Thesis. Fujian Medical University. 2013 (In Chinese).

30. Zhang C: Vaccine of target gene MTDH/AEG-1 induced prostate cancer growth, metastasis and enhanced chemotherapy sensitivity to paclitaxel. Master Thesis. Fujian Medical University. 2012 (In Chinese)

31. Nikpour M, Emadi-Baygi M, Fischer U, et al: MTDH/AEG-1 contributes to central features of the neoplastic phenotype in bladder cancer. Urol Oncol 32: 670-676, 2014.

32. Chen W, Ke Z, Shi H, Yang S and Wang L: Overexpression of AEG-1 in renal cell carcinoma and its correlation with tumor nuclear grade and progression. Neoplasma 57: 522-529, 2010.

33. Erdem H, Oktay M, Yildirim U, Uzunlar AK and Kayikci MA: Expression of AEG-1 and p53 and their clinicopathological significance in malignant lesions of renal cell carcinomas: a microarray study. Pol J Pathol 64: 28-32, 2013.

34. Wu C, Jin B, Chen L, Zhuo D, Zhang Z, Gong K and Mao Z: MiR-30d induces apoptosis and is regulated by the Akt/FOXO pathway in renal cell carcinoma. Cell Signal 25: 1212-1221, 2013. 